\title{
Spectroscopic Evaluation of Thermodynamic Parameters for Aluminum Based Diatomic Gas Molecules
}

\author{
P. Sriramachandran ${ }^{1 *}$, B. Viswanathan ${ }^{2}$ and R. Shanmugavel ${ }^{1}$ \\ ${ }^{1}$ Physics Research Center, VHNSN College, Virudhunagar - 626001 , India. \\ ${ }^{2}$ Department of physics, Sri Kaliswari college, Sivakasi - 626 123, India. \\ sri_ramnivash@rediffmail.com, rsvel67@gmail.com
}

\begin{abstract}
On account of the high accuracy with which spectroscopic data are obtained, thermodynamic parameters estimated from these data are more reliable than those obtained by direct thermal measurements. Spectroscopic data of aluminum based diatomic gas molecules $(\mathrm{AlO}, \mathrm{AlH}, \mathrm{AlD}, \mathrm{AlF}, \mathrm{AlC})$ and partition function theory have been used to evaluate the thermodynamical parameters in the temperature range $100-3000{ }^{0} \mathrm{~K}$. Equilibrium constants for astrophysically important chemical process have been calculated using partition function theory. It is noticed that for AlC the heat capacity decreases exponentially after the critical temperature $1200{ }^{0} \mathrm{~K}$.
\end{abstract}

\section{Keywords: Diatomic molecule; partition function; entropy; equilibrium constant.}

\section{Introduction}

If we can assume chemical equilibrium, then the abundance of a molecule is determined independent of its formation mechanism, and therefore the most important factor determining the thermodynamical stability of the molecule is the dissociation energy. The molecules in stellar atmospheres can be reasonably well understood on the basis of chemical equilibrium theory [1]. Thermodynamical parameters (such as free energy, enthalpy, entropy and heat capacity etc.) of diatomic molecules calculated experimentally are not accurate and sometimes such measurement is not possible. These properties have been useful in the fabrication of smart devices using intelligent materials $[2,3]$. These parameters have also been used in medical field [4]. Spectroscopic evaluation of thermodynamical parameters was first suggested by Tolman [5] using prior probability. Sauval and Tatum [6] have used the spectroscopic properties of high temperature data set to evaluate the polynomial expressions of partition functions and equilibrium constants for 300 molecules of astrophysical interest. Cardelino et al [7] have reported molecular thermodynamical parameters for indium compound. Hildenbrand and Lau [8] have determined the dissociation energies of copper and silver mono halides and nickel mono fluorides using thermodynamical parameters from the method of quantum chemical calculation. Most recently thermo dynamical parameters of tin chalcogenide [9] and platinum diatomic molecule [10] have been reported by statistical thermodynamics method using spectroscopy constants.

Thermodynamical data can be used for the estimation of equilibrium constants of various astro-chemical reactions. The thermodynamical study of metallic hydrides and deuterides is of astrophysical importance. Viswanathan et al [11] have identified the $\mathrm{CrH}$ and $\mathrm{CrD}$ molecular lines in sunspot umbral spectrum. Pavlenko et al [12] have reported equilibrium studies of $\mathrm{MgH}, \mathrm{MgD}, \mathrm{CrH}$ and $\mathrm{CrD}$ molecules. These studies are used for the deuterium test. Aluminum Monoxide ( $\mathrm{AlO})$ is an important gas phase combustion intermediate of aluminum with oxygen containing oxidizers, such as $\mathrm{H}_{2} \mathrm{O}, \mathrm{CO}_{2}$, and $\mathrm{O}_{2}$. Typically, it is seen in emission and absorption during combustion of aluminum particles at temperatures greater than $2300{ }^{0} \mathrm{~K}$. Swings [13] has reported the occurrence of AlO bands in stellar sources. Aluminum, the $12^{\text {th }}$ most abundant element in space, is the most refractive of the 20 leading elements in the Periodic Table [14]. This high degree of refractivity translates into substantial interstellar depletions of gasphase aluminum; in diffuse clouds, for example, 90\%-99\% of this element is found to be depleted into grains [15]. Despite the propensity of aluminum to condense, the element exhibits significant gas phase chemistry in carbonrich circumstellar environments. Gas-phase thermodynamic equilibrium chemistry is the likely formation mechanism for the detection of $\mathrm{AlO}$ in $\mathrm{VY} \mathrm{CMa} \mathrm{[16].} \mathrm{Also} \mathrm{the}$ detection of $\mathrm{AlO}$ is an evidence of an active gas-phase refractory chemistry in oxygen-rich envelopes. To the best of our knowledge, there has been no report so far in the literature on the thermo dynamical parameters of $\mathrm{Al}$ based diatomic molecules. Hence we have evaluated thermo dynamical parameters of $\mathrm{AlO}, \mathrm{AlH}, \mathrm{AlD}, \mathrm{AlF}$ and $\mathrm{AlC}$ molecules by partition function theory, using the spectroscopic constants. The molecular constants used in this study were collected form the literature and are listed in Table 1. The astro-chemical reactions are also described in the following section and their equilibrium constants are listed in the respective table.

\section{Partition Function and Thermodynamic Parameters}

In the case of thermochemical non-equilibrium gas, multiple temperatures are defined to describe the partitioning of energy across the internal modes. For example, translational, rotational, vibrational, and electronic energy modes for the mixture or for each species may be used. The various states of each energy mode are assumed to be in equilibrium at the characteristic temperature. Each mode is described by its own energy conservation equation which includes terms coupling to the 
other energy modes. Usually, separate internal modes for each species are not defined because the energy modes are assumed to be strongly coupled between species. Therefore the total internal energy of a diatomic molecule is given by

$$
E_{T}=E_{r o t}+E_{v i b}+E_{\text {ele }}+E_{\text {tran }}
$$

To evaluate the partition function, it is necessary to know all the structural data (such as rotational, vibrational, electronic and translational) of each molecule. Therefore the total partition function of a molecular system can be expressed as:

$$
Z_{T}=Z_{r o t} \times Z_{v i b} \times Z_{\text {ele }} \times Z_{\text {tran }}
$$

In calculating the thermal parameters for high temperatures, the low-lying electronic states of the molecule should be considered. The different multiplet components of the lowest electronic states can be taken into account by calculating the electronic partition function $Z_{\text {ele }}$ in "Eq. (2)". This is not possible for electronic states that have different rotational and vibrational constants from the ground state. Rather an additional term corresponding to all the rotational and vibrational levels of the excited electronic state has to be added to the partition function for the ground state including the non-rigidity, anharmonicity and stretching effect. Thus the total partition function can be expressed as:

$$
Z_{T}=Z_{\text {tran }} Z_{v r}
$$

The translational partition function is given by

$$
\frac{Z_{\text {tran }}}{N}=\left(\frac{2 \pi m k T}{h^{2}}\right)^{3 / 2} \frac{k T}{P_{0}}
$$

where $m, N, k, h, \quad T$ and $P_{0}\left(=1.01325 \times 10^{5} \mathrm{Nm}^{-2} \mathrm{~atm}^{-1}\right)$ represent the mass of the molecule, total number of molecules, Boltzmann's constant, Planck's constant, absolute temperature in Kelvin and standard state of one atmospheric pressure respectively. The translational part can always be separated and its contribution to the thermodynamical parameters can be calculated using following relations

Free energy $F_{\text {tran }}=-\frac{3}{2} R T \ln m-\frac{5}{2} R T \ln T+6.697 R T$

Enthalpy $H_{\text {tran }}=\frac{5}{2} R T$

Entropy $S_{\text {tran }}=\frac{5}{2} R+\frac{F_{\text {tran }}}{T}$

Heat capacity at constant pressure $C_{P_{\text {ran }}}=\frac{5}{2} R$

For diatomic species the internal partition function includes vibrational and rotational energies,

$Z_{v r}=\frac{1}{\sigma} \sum_{v=0}^{v_{\max }} \sum_{J=0}^{J_{\max }} g(v, J) e^{-\frac{E(v, J)}{k T}}$ where $v$ and $J$ are the vibrational and rotational quantum numbers respectively and $\sigma$ is the symmetry number.

The total internal energy of diatomic molecule is given by

$$
\begin{array}{r}
E(v, J)=\left(\omega_{e}(v+1 / 2)-\omega_{e} x_{e}(v+1 / 2)^{2}+B_{e} J(J+1)-\right. \\
\left.D_{e} J^{2}(J+1)^{2}-\alpha_{e}(v+1 / 2) J(J+1)\right)
\end{array}
$$

which is useful for values $\mathrm{v}$ and $\mathrm{J}$ that significantly contribute to thermodynamic properties. Neglecting the zero point vibrational energy [17], an equation equivalent to "Eq. (6)" that gives the energy above the ground state ( $\mathrm{v}=$ $0, \mathbf{J}=0$ ) can be written as follows,

$$
\begin{aligned}
E(v, J)=\omega_{0} v-\omega_{0} x_{0} v(v-1) & +J(J+1) \\
& {\left[B_{0}-D_{e} J(J+1)-\alpha_{e} v\right] }
\end{aligned}
$$

where $B_{0}=B_{e}-\frac{\alpha_{e}}{2}$;

$\omega_{0}=\omega_{e}-2 \omega_{e} x_{e} ; \omega_{0} x_{0}=\omega_{e} x_{e}$

Further substitution for simplification,

$u=\frac{h c \omega_{0}}{k T} ; y=\frac{h c B_{0}}{k T} ; \beta=\frac{D_{e}}{B_{0}} ; \delta=\frac{\alpha_{e}}{B_{0}}$

and $D_{e}=\frac{4 B_{e}^{3}}{\omega_{e}^{2}}$ gives:

$$
\begin{array}{r}
E(v, J)=u v(1-x(v-1)+J(J+1) y \\
{[1-\beta J(J+1)-v \delta]}
\end{array}
$$

Then the vibration rotation partition function "Eq. (5)" can be simplified as

$Z_{v r}=\frac{1}{\sigma \mathrm{y}\left(1-\mathrm{e}^{-\mathrm{u}}\right)}\left[1+\frac{2 \beta}{\mathrm{y}}+\frac{\delta}{e^{u}-1}+\frac{2 x u}{\left(e^{u}-1\right)^{2}}+\frac{y}{3}+\frac{y^{2}}{15}\right]$

here $\sigma$ is the symmetry number and its value is 1 for hetronuclear molecule and 2 for homonuclear molecule and $\mathrm{x}$ is called the multiplicity of the electronic ground state of the molecule. Thus the vibration rotation contribution to the thermodynamical parameters can be calculated using following relations.

Free energy $F_{v r}=-R T \ln Z_{v r}$

Enthalpy $H_{v r}=R T^{2} \frac{\partial}{\partial T}\left(\ln Z_{v r}\right)$

Entropy $S_{v r}=R\left[\left(1+\ln Z_{v r}\right)\right]+R T\left[\frac{\partial}{\partial T}\left(\ln Z_{v r}\right)\right]$

Heat capacity at constant pressure

$C_{P_{v r}}=R T \frac{\partial}{\partial T}\left(\ln Z_{v r}\right)$

The sum of the translational and vibrational-rotational contributions gives the final value of the thermodynamical parameters. 


\section{Equilibrium Constants}

Computations of chemical equilibria have been used directly for interpretations of observed molecular features in red giant stars [18] and also it can be used for the deuterium test. In general the chemical process involving the two reactants and two products is represented by

$$
A+B \Leftrightarrow C+D
$$

Then the equilibrium constant $K$ is given by

$$
K=\frac{Z(C) Z(D)}{Z(A) Z(B)} \times e^{-\left(\frac{\Delta E}{k T}\right)}
$$

Here $\Delta E$ is the difference between zero point energies of the diatomic molecules of relative abundance. $Z$ is the total partition functions which can be factorized into the internal and translational components.

We have calculated the equilibrium constants for the following three reactions of astrophysical interest in the temperature range $100-3000 \mathrm{~K}$.

$$
A l H+H D \Leftrightarrow A l D+H_{2}
$$

$A l D+H D \Leftrightarrow A l H+D_{2}$

$A l H+O D \Leftrightarrow A l D+O H$

Almost in all the astrophysical sources, hydrogen and deuterium are primarily found in their molecular form. It is worth to study the chemical processes involving diatomic metallic hydrides and deuterides when found present in the astrophysical sources. The equilibrium constants are listed in Table 4. The equilibrium constants indicate that at low temperatures the formation of the deuterides are strongly favoured over the hydrides, but at high temperatures the hydrides become weakly favoured. It is worth noting that in cool dwarfs hydrogen and deuterium are primarily found in their molecular forms, $\mathrm{H}_{2}, \mathrm{HD}$ and $\mathrm{D}_{2}$ [12]. In a complete chemical equilibrium calculation the partition functions and dissociation energies of $\mathrm{H}_{2}, \mathrm{HD}$ and $\mathrm{D}_{2}$ will have an effect upon the abundances of the molecules of interest.

\section{Results and Discussions}

The estimated thermodynamical parameters are listed in Table 2 to 6 . Results of the study show that free energy, enthalpy and entropy increase with increase in the temperature. Heat capacity also increases with temperature except for Aluminum Carbide (AlC). In AlC, heat capacity $\left(C_{P}\right)$ increases up to critical temperature $1200{ }^{0} \mathrm{~K}$ and then decreases. This is may be due to interaction of the molecule to its metastable state and to spin reorientation because of high multiplicity in ground state. A similar phenomenon is found in polyatomic molecules which involves hindered rotation [17]. Also this may be due to formation of ions of the molecule at such high temperature. Similar behavior is noticed in $\mathrm{SiC}$ at $2300{ }^{0} \mathrm{~K}$ and in $\mathrm{CrC}$ at $1900{ }^{0} \mathrm{~K}$, the graphical representation of such variations is shown in "Fig. 1 ". In general it is noticed that all the diatomic carbides, having high ground state multiplicity exhibit such phenomena. Increase in entropy of the molecules shows that the disorder of the system is high at high temperatures. The equilibrium constant of the astrophysically important reaction in the temperature range $100-3000{ }^{0} \mathrm{~K}$ are listed in the Table 7. Graphical representation of equilibrium constant versus temperature is shown in "Fig. 2". The study shows that the change in equilibrium constant goes on decreasing and becomes almost constant after a certain temperature. At low temperatures only translational part of motion gives its contribution but as temperature increases rotational, vibrational and electronic motions are excited. Enthalpy represents the thermal energy of any system and depends on internal energy, pressure and volume. Therefore the internal energy of an ideal gas depends only on its temperature. Molecules are said to be in thermal motion as a result of their being at a particular temperature, and their thermal motion increases with increased temperature. Therefore the molecules in a system at high temperature are highly distributed either in terms of their locations or in terms of occupation of their possible internal modes of energy states, such as rotation and vibration. The free energy is expressed in terms representing the translation and internal degrees of freedom. Internal energy and entropy are functions of temperature and hence increase with temperature.

\section{Conclusions}

Due to the involvement of spectroscopic data the estimated thermodynamic parameters are highly accurate; such measurement is not possible experimentally at high temperature. The equilibrium constant for the astrochemical processes is applicable to the deuterium test. Since silicon carbide is thought to be an important component of the dust shells surrounding the carbon rich stars [19,20]. The observation of $\mathrm{SiC}$ in the dust shell of carbon stars suggests that the metal carbides such as $\mathrm{FeC}$ [21], $\mathrm{AlC}, \mathrm{CrC}$ and all diatomic carbides having high ground state multiplicity may be present as well. These data may be useful for chemical and electronic industries where the performance of the devices is affected by the thermodynamic parameters.

\section{References}

[1] H. N. Russell, Molecules in the Sun and Stars, Astrophys. J., 79, 317-342, 1934.

[2] K. Sadeghipour, R. Solamon, S. Neogi, Development of a novel electrochemically active membrane and 'smart' material based vibration sensor/damper, Smart. Mater. Struct., 1, 172-179, 1992.

[3] P. Kotzian, P. Brazdilova, K. Kalcher, K. Handlir, K. Vytras, Oxides of platinum metal group as potential catalysts in carbonaceous amperometric biosensors based on oxidases, Sens. Actuators B. Chem., 124, 297 302, 2006.

[4] D. R. Chialvo, Physiology: Unhealthy surprises, Nature, 419, 263-264, 2002.

[5] R. C. Tolman, Rotational Specific Heat and Half Quantum Numbers, Phys. Rev., 22, 470-478, 1923.

[6] A. J. Sauval, J. B. Tatum, A Set of Partition Functions and Equilibrium Constants for 300 Diatomic Molecules of Astrophysical Interest, Astrophys. J. Sup. Ser., 56, 193-209, 1984.

[7] B. H. Cardelino, C. E. Moore, C. A. Cardelinp, D. O. Frazier, K. J. Bechmann, Theoretical Study of Indium Compounds of Interest for Organometallic Chemical Vapour Deposition. J. Phys. Chem. A, 105, 849-868, 2001. 
[8] D. L. Hildenbrand, K. H. Lau, Dissociation energies of the $\mathrm{Cu}$ and $\mathrm{Ag}$ monohalides and of Ni monofluoride, $J$. Phys. Chem. A, 110, 11886-11889, 2006.

[9] Pavitra Tandon., K. N. Uttam, Estimation of thermodynamics quantities of tin chalcogenides from spectroscopic data, Indian J. Pure and App. Phys., 47, 725-729, 2009.

[10] Pavitra Tandon., K. N. Uttam, Thermodynamic properties of Platimum Diatomics: Properties of $\mathrm{PtH}$, $\mathrm{PtC}, \mathrm{PtN}$, and $\mathrm{PtO}$ calculated from spectroscopic data, Platinum Metal Review, 53,123-134, 2009.

[11] B. Viswanathan, R. Shanmugavel, S. P. Bagare, N. Rajamanickam, P. Sriramachandran, Identification of $\mathrm{CrH}$ and CrD Molecular Lines in the Sunspot Umbral Spectrum, Solar Phys., 257,261-269, 2009.

[12] Ya. V. Pavlenko, G. J. Harris, J. Tennyson, H. R. A. Jones, J. M. Brown, C. Hill, L. A. Yakovina, The electronic bands of $\mathrm{CrD}, \mathrm{CrH}, \mathrm{MgD}$ and $\mathrm{MgH}$ : application to the 'deuterium test', Mon. Not. Rom. Astrom. Soc., 386, 1338-1346, 2008.

[13] P. Swings, Spectroscopic problems of astronomical interest, J. Opt. Soc. Amer., 41, 153-165, 1951.

[14] K. Lodders, Solar system abundances and condensation temperatures of the elements, Astrophys. J., 591, 1220-1247, 2003.

[15] B. E. Turner, A molecular line survey of Sagittarius B2 and Orion-KL from 70 to $115 \mathrm{GHz}$. II - Analysis of the data, Astophys. J. Sup. Ser., 76, 617-686, 1991.

[16] E. D. Tenenbaum, L. M. Ziurys, Millimeter Detection

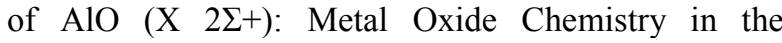
Envelope of VY Canis Majoris, Astrophys. J., 694, L59-L63, 2009.

[17] J. Bevan Ott, Juliana Boerio-Goates, Chemical Thermodynamics: Principles and Applications, Elsevier Academic Press, San Diego, California, USA, 2000.

[18] J. M. Scalo, Studies of evolved stars. II. On the interpretation of the $\mathrm{CN} / \mathrm{C}_{2}$ and $\mathrm{CO} / \mathrm{C}_{2}$ ratios in cool carbon stars, Astrophys. J., 184, 801-813, 1973.

[19] A.T. Barrett, On the microwave detection of interstellar $\mathrm{CH}_{4}, \mathrm{SiH}_{4}$, and $\mathrm{GeH}_{4}$. Astrophys. J., 220, L81-L85, 1978.

[20] D. M. Goldhaber, A. L. Betz, Silane in IRC +10216, Astrophys. J., 279, L55-L58, 1984.

[21] M. D. Allen, T. C. Pesch, S. Ziury, The Pure Rotational Spectrum of FeC $\left(X^{3} \Delta_{i}\right)$, Astrophys. J., 472, L57-L60, 1996.

[22] W. Szajana, M. Zachwieja, Emission spectroscopy of the $\mathrm{A}^{1} \Pi-\mathrm{X}^{1} \Sigma^{+}$system of AlH, Eur. Phys. J. D, 55, $549-555,2009$

[23] J. B. White, M. Dulick, P. F. Bernath, High resolution infrared emission spectra of $\mathrm{AlH}$ and AlD, J. Chem. Phys., 99, 8371-8378, 1993.

[24] R. B. Christopher, A. T. Mickelle, The electronic emission spectrum of aluminum carbide, J. Mol. Spectrosc, 258, 42-49, 2009.

[25] M. D. Saksena, M. N. Deo, K. Sunanda, S. H. Behere, C. T. Londe, Fourier transform spectral study of $\mathrm{B}^{2} \Sigma^{+}$$\mathrm{X}^{2} \Sigma^{+}$system of AlO, J. Mol. Spectrosc., 247, 47-56, 2008 .
[26] R. F. Barrow, I. Kopp, C. Malmberg, The Electronic Spectrum of Gaseous AIF, Physica Scripta, 10, 86-102, 1974.

[27] R. A. Durie, G. Herzberg, Forbidden transitions in diatomic molecules. $\mathrm{V}$. The rotation-vibration spectrum of the hydrogen-deuteride (HD) molecule, Can. J. Phys., 38, 806-818, 1960.

[28] A.R. W. McKellar, W. Goetz, D. A. Ramsay, The rotation-vibration spectrum of HD - Wavelength and intensity measurements of the 3-0, 4-0, 5-0, and 6-0 electric dipole bands, Astrophys. J., 207, 663-670, 1976.

[29] M. Mizushima, Molecular Parameters of OH Free Radical, Phys. Rev. A: Gen. Phys., 5, 143-157, 1972.

[30] G. C. Dousmanis, T. M. Sanders, C. H. Townes, Microwave Spectra of the Free Radicals OH and OD, Phys. Rev., 100, 1735-1754, 1955.

[31] G. Herzberg, Spectra of diatomic molecules, $2^{\text {nd }} E d$., New York, Nastrand Reinhold, 1950.

[32] P. J. Brannon, C. H. Church, C. W. Peters, Electric field induced spectra of molecular hydrogen, deuterium and deuterium hydride, J. Mol. Spectrosc., 27, 44-54, 1968 .

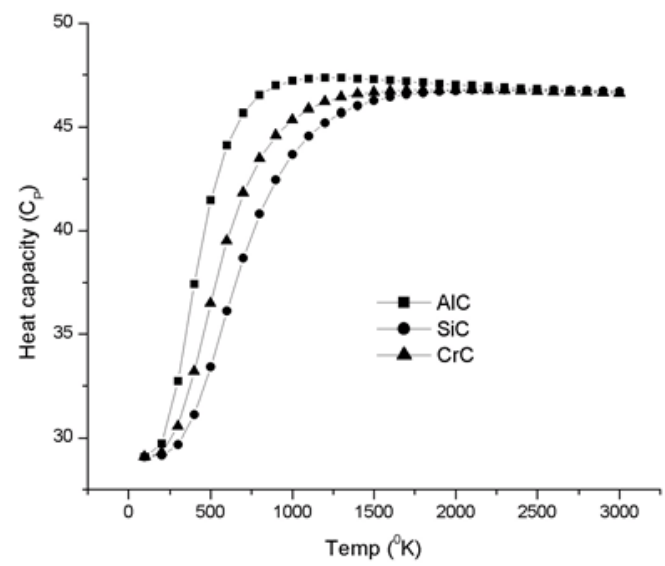

Fig. 1. Temperature versus Heat Capacity at constant Pressure.

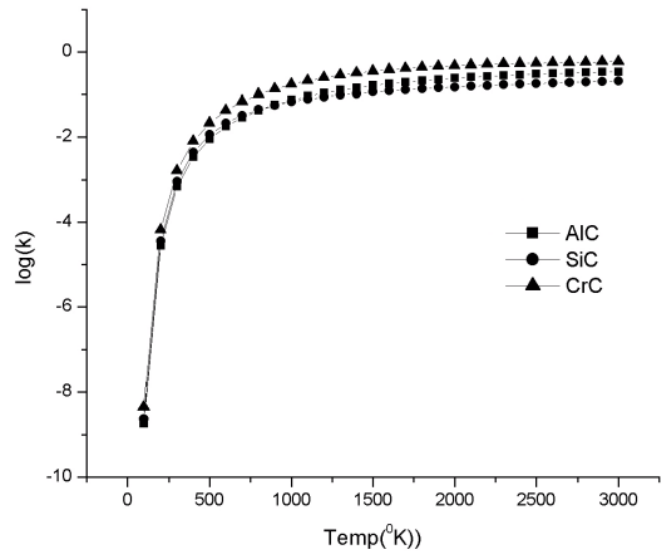

Fig. 2. Temperature versus logarithmic value of equilibrium constant. 
Table 1. Spectroscopic Constants of Gas Phase Diatomic Molecules.

\begin{tabular}{lccccccc}
\hline Mol. & $x$ & $\begin{array}{c}\omega_{e} \\
\left(\mathrm{~cm}^{-1}\right)\end{array}$ & $\begin{array}{c}\omega_{e} x_{e} \\
\left(\mathrm{~cm}^{-1}\right)\end{array}$ & $\begin{array}{c}B_{e} \\
\left(\mathrm{~cm}^{-1}\right)\end{array}$ & $\begin{array}{c}\alpha_{e} \\
\left(\mathrm{~cm}^{-1}\right)\end{array}$ & $\begin{array}{c}M W \\
(\mathrm{amu})\end{array}$ & Ref. \\
\hline $\mathrm{AlH}$ & 1 & 1682.374 & 29.051 & 6.393 & 0.187 & 27.989 & {$[22]$} \\
$\mathrm{AlD}$ & 1 & 1211.774 & 15.064 & 3.318 & 0.069 & 28.995 & {$[23]$} \\
$\mathrm{AlC}$ & 4 & 654.893 & 4.347 & 0.531 & 0.005 & 38.992 & {$[24]$} \\
$\mathrm{AlO}$ & 2 & 979.524 & 7.036 & 0.641 & 0.005 & 42.980 & {$[25]$} \\
$\mathrm{AlF}$ & 1 & 802.260 & 4.770 & 0.552 & 0.004 & 4.598 & {$[26]$} \\
$\mathrm{HD}$ & 1 & 3813.100 & 91.650 & 45.655 & 1.986 & 3.022 & {$[27]$} \\
$\mathrm{OH}$ & 2 & 3737.760 & 84.881 & 18.910 & 0.724 & 17.007 & {$[29]$} \\
$\mathrm{OD}$ & 2 & 2720.240 & 44.055 & 10.020 & 0.275 & 18.013 & {$[30]$} \\
$\mathrm{H}$ & 1 & 4401.210 & 121.330 & 60.853 & 3.062 & 2.015 & {$[31]$} \\
$\mathrm{D}_{2}$ & 1 & 3115.500 & 61.820 & 30.443 & 1.078 & 4.028 & {$[32]$} \\
\hline
\end{tabular}

Table 2. Calculated Thermodynamic Properties of AlO Molecule

\begin{tabular}{ccccc}
\hline$T\left(^{0} K\right)$ & $F$ & $H$ & $S$ & $C_{p}$ \\
\hline 100 & -12.589 & 2.908 & 163.28 & 29.078 \\
200 & -29.211 & 5.830 & 183.522 & 29.151 \\
300 & -47.383 & 8.880 & 195.856 & 29.600 \\
400 & -66.638 & 12.296 & 205.65 & 30.74 \\
500 & -86.819 & 16.264 & 214.48 & 32.528 \\
600 & -107.852 & 20.783 & 222.706 & 34.639 \\
700 & -129.676 & 25.717 & 230.304 & 36.738 \\
800 & -152.226 & 30.891 & 237.211 & 38.614 \\
900 & -175.432 & 36.166 & 243.424 & 40.185 \\
1000 & -199.227 & 41.452 & 248.993 & 41.452 \\
1100 & -223.549 & 46.698 & 253.994 & 42.453 \\
1200 & -248.347 & 51.883 & 258.506 & 43.236 \\
1300 & -273.574 & 57.002 & 262.603 & 43.847 \\
1400 & -299.193 & 62.055 & 266.349 & 44.325 \\
1500 & -325.171 & 67.050 & 269.795 & 44.700 \\
1600 & -351.480 & 71.993 & 272.985 & 44.995 \\
1700 & -378.097 & 76.890 & 275.954 & 45.229 \\
1800 & -405.002 & 81.748 & 278.731 & 45.415 \\
1900 & -432.175 & 86.572 & 281.339 & 45.564 \\
2000 & -459.602 & 91.368 & 283.799 & 45.684 \\
2100 & -487.267 & 96.139 & 286.127 & 45.780 \\
2200 & -515.160 & 100.888 & 288.336 & 45.858 \\
2300 & -543.268 & 105.619 & 290.439 & 45.921 \\
2400 & -571.582 & 110.335 & 292.446 & 45.973 \\
2500 & -600.092 & 115.036 & 294.366 & 46.014 \\
2600 & -628.790 & 119.726 & 296.205 & 46.048 \\
2700 & -657.667 & 124.404 & 297.971 & 46.076 \\
2800 & -686.718 & 129.074 & 299.669 & 46.098 \\
2900 & -715.936 & 133.735 & 301.305 & 46.116 \\
3000 & -745.315 & 138.389 & 302.882 & 46.130 \\
\hline & & & &
\end{tabular}

Table 3. Calculated Thermodynamic Properties of AlH Molecule.

\begin{tabular}{ccccc}
\hline$T\left({ }^{0} K\right)$ & $F$ & $H$ & $S$ & $C_{p}$ \\
\hline 100 & -10.182 & 2.977 & 138.991 & 28.859 \\
200 & -24.375 & 5.891 & 159.185 & 28.997 \\
300 & -40.094 & 8.814 & 171.036 & 29.077 \\
400 & -56.812 & 11.785 & 179.578 & 29.234 \\
500 & -74.291 & 14.860 & 186.434 & 29.538 \\
600 & -92.404 & 18.095 & 192.327 & 30.006 \\
700 & -111.073 & 21.529 & 197.616 & 30.626
\end{tabular}

\begin{tabular}{ccccc}
800 & -130.250 & 25.183 & 202.493 & 31.365 \\
900 & -149.899 & 29.058 & 207.054 & 32.186 \\
1000 & -169.990 & 33.140 & 211.353 & 33.049 \\
1100 & -190.499 & 37.405 & 215.417 & 33.922 \\
1200 & -211.403 & 41.828 & 219.264 & 34.780 \\
1300 & -232.682 & 46.379 & 222.906 & 35.606 \\
1400 & -254.315 & 51.033 & 226.355 & 36.387 \\
1500 & -276.284 & 55.766 & 229.620 & 37.117 \\
1600 & -298.570 & 60.559 & 232.713 & 37.792 \\
1700 & -321.158 & 65.395 & 235.645 & 38.414 \\
1800 & -344.031 & 70.260 & 238.426 & 38.983 \\
1900 & -367.176 & 75.144 & 241.066 & 39.502 \\
2000 & -390.577 & 80.039 & 243.577 & 39.974 \\
2100 & -414.224 & 84.938 & 245.967 & 40.403 \\
2200 & -438.104 & 89.837 & 248.246 & 40.794 \\
2300 & -462.207 & 94.732 & 250.422 & 41.148 \\
2400 & -486.523 & 99.620 & 252.502 & 41.470 \\
2500 & -511.042 & 104.499 & 254.494 & 41.763 \\
2600 & -535.756 & 109.369 & 256.404 & 42.030 \\
2700 & -560.657 & 114.229 & 258.238 & 42.273 \\
2800 & -585.738 & 119.078 & 260.002 & 42.495 \\
2900 & -610.993 & 123.916 & 261.700 & 42.698 \\
3000 & -636.413 & 128.743 & 263.336 & 42.884 \\
\hline & & & &
\end{tabular}

Table 4. Calculated Thermodynamic Properties of AlD Molecule.

\begin{tabular}{ccccc}
\hline$T\left({ }^{0} K\right)$ & $F$ & $H$ & $S$ & $C_{p}$ \\
\hline 100 & -10.756 & 2.989 & 144.851 & 28.98 \\
200 & -25.535 & 5.905 & 165.06 & 29.071 \\
300 & -41.847 & 8.871 & 177.073 & 29.267 \\
400 & -59.187 & 11.989 & 186.027 & 29.744 \\
500 & -77.342 & 15.353 & 193.522 & 30.523 \\
600 & -96.202 & 19.014 & 200.189 & 31.539 \\
700 & -115.698 & 22.975 & 206.288 & 32.691 \\
800 & -135.781 & 27.201 & 211.928 & 33.887 \\
900 & -156.407 & 31.644 & 217.159 & 35.058 \\
1000 & -177.537 & 36.251 & 222.012 & 36.16 \\
1100 & -199.135 & 40.978 & 226.516 & 37.169 \\
1200 & -221.167 & 45.784 & 230.697 & 38.077 \\
1300 & -243.602 & 50.641 & 234.585 & 38.884 \\
1400 & -266.412 & 55.526 & 238.205 & 39.596 \\
1500 & -289.572 & 60.424 & 241.584 & 40.222 \\
1600 & -313.059 & 65.325 & 244.747 & 40.771 \\
1700 & -336.852 & 70.22 & 247.715 & 41.252 \\
1800 & -360.933 & 75.105 & 250.507 & 41.674 \\
1900 & -385.285 & 79.977 & 253.142 & 42.045 \\
2000 & -409.894 & 84.836 & 255.634 & 42.372 \\
2100 & -434.745 & 89.679 & 257.997 & 42.661 \\
2200 & -459.826 & 94.508 & 260.243 & 42.917 \\
2300 & -485.127 & 99.322 & 262.383 & 43.144 \\
2400 & -510.637 & 104.121 & 264.426 & 43.346 \\
2500 & -536.346 & 108.908 & 266.38 & 43.527 \\
2600 & -562.247 & 113.681 & 268.252 & 43.688 \\
2700 & -588.331 & 118.443 & 270.049 & 43.834 \\
2800 & -614.592 & 123.193 & 271.776 & 43.965 \\
2900 & -641.022 & 127.933 & 273.44 & 44.084 \\
3000 & -667.615 & 132.664 & 275.044 & 44.191 \\
\hline & & & &
\end{tabular}


Table 5. Calculated Thermodynamic Properties of AlC Molecule.

\begin{tabular}{ccccc}
\hline$T\left({ }^{0} K\right)$ & $F$ & $H$ & $S$ & $C_{p}$ \\
\hline 100 & -12.632 & 3.000 & 163.731 & 29.092 \\
200 & -29.320 & 6.035 & 184.634 & 29.720 \\
300 & -47.737 & 9.908 & 200.162 & 32.722 \\
400 & -67.668 & 15.053 & 214.889 & 37.404 \\
500 & -88.989 & 20.827 & 227.763 & 41.471 \\
600 & -111.477 & 26.571 & 238.242 & 44.133 \\
700 & -134.908 & 32.065 & 246.716 & 45.677 \\
800 & -159.109 & 37.314 & 253.729 & 46.528 \\
900 & -183.956 & 42.377 & 259.694 & 46.984 \\
1000 & -209.359 & 47.308 & 264.890 & 47.217 \\
1100 & -235.252 & 52.147 & 269.503 & 47.323 \\
1200 & -261.582 & 56.921 & 273.657 & 47.358 \\
1300 & -288.308 & 61.649 & 277.442 & 47.352 \\
1400 & -315.397 & 66.343 & 280.920 & 47.323 \\
1500 & -342.821 & 71.012 & 284.142 & 47.281 \\
1600 & -370.555 & 75.663 & 287.144 & 47.232 \\
1700 & -398.580 & 80.299 & 289.955 & 47.181 \\
1800 & -426.878 & 84.925 & 292.598 & 47.130 \\
1900 & -455.432 & 89.542 & 295.095 & 47.079 \\
2000 & -484.229 & 94.151 & 297.459 & 47.030 \\
2100 & -513.257 & 98.756 & 299.705 & 46.983 \\
2200 & -542.504 & 103.355 & 301.845 & 46.938 \\
2300 & -571.960 & 107.951 & 303.888 & 46.896 \\
2400 & -601.616 & 112.544 & 305.843 & 46.855 \\
2500 & -631.463 & 117.135 & 307.717 & 46.817 \\
2600 & -661.494 & 121.723 & 309.516 & 46.781 \\
2700 & -691.701 & 126.309 & 311.247 & 46.747 \\
2800 & -722.078 & 130.894 & 312.915 & 46.715 \\
2900 & -752.619 & 135.478 & 314.523 & 46.685 \\
3000 & -783.318 & 140.06 & 316.077 & 46.656 \\
\hline & & & &
\end{tabular}

Table 6. Calculated Thermodynamic Properties of AlF Molecule.

\begin{tabular}{ccccc}
\hline$T\left({ }^{0} K\right)$ & $F$ & $H$ & $S$ & $C_{p}$ \\
\hline 100 & -12.805 & 3.000 & 165.445 & 29.084 \\
200 & -29.648 & 5.945 & 185.823 & 29.269 \\
300 & -48.070 & 9.108 & 198.603 & 30.056 \\
400 & -67.624 & 12.674 & 208.83 & 31.457 \\
500 & -88.13 & 16.681 & 217.755 & 33.18 \\
600 & -109.48 & 21.054 & 225.719 & 34.938 \\
700 & -131.584 & 25.681 & 232.848 & 36.557 \\
800 & -154.362 & 30.462 & 239.23 & 37.964 \\
900 & -177.745 & 35.325 & 244.957 & 39.149 \\
1000 & -201.671 & 40.222 & 250.117 & 40.131 \\
1100 & -226.089 & 45.124 & 254.789 & 40.939 \\
1200 & -250.953 & 50.017 & 259.046 & 41.605 \\
1300 & -276.224 & 54.891 & 262.948 & 42.154 \\
1400 & -301.869 & 59.744 & 266.544 & 42.609 \\
1500 & -327.861 & 64.574 & 269.877 & 42.988 \\
1600 & -354.174 & 69.382 & 272.98 & 43.307 \\
1700 & -380.787 & 74.170 & 275.883 & 43.576 \\
1800 & -407.682 & 78.940 & 278.609 & 43.805 \\
1900 & -434.841 & 83.692 & 281.178 & 44.000 \\
2000 & -462.25 & 88.429 & 283.608 & 44.169 \\
2100 & -489.895 & 93.152 & 285.913 & 44.315
\end{tabular}

\begin{tabular}{lcccc}
2200 & -517.766 & 97.863 & 288.104 & 44.442 \\
2300 & -545.85 & 102.562 & 290.193 & 44.553 \\
2400 & -574.138 & 107.252 & 292.189 & 44.65 \\
2500 & -602.622 & 111.933 & 294.1 & 44.737 \\
2600 & -631.293 & 116.606 & 295.933 & 44.813 \\
2700 & -660.143 & 121.271 & 297.693 & 44.881 \\
2800 & -689.166 & 125.930 & 299.388 & 44.942 \\
2900 & -718.356 & 130.583 & 301.021 & 44.997 \\
3000 & -747.706 & 135.231 & 302.596 & 45.047 \\
\hline
\end{tabular}

Note: In Tables $1-6$,

$T$ - Temperature $\left({ }^{0} \mathrm{~K}\right)$

$F$ - Free energy (KJ/mole)

$H$ - Enthalpy (KJ/mole),

$S$ - Entropy (KJ/mole)

$C_{P}$ - Heat capacity $\left(J / K_{\text {mole }}\right)$

Table 7. Equilibrium Constants of Reactions.

\begin{tabular}{cccc}
\hline$T\left({ }^{0} K\right)$ & {$[a]$} & {$[b]$} & {$[c]$} \\
\hline 100 & $1.87 \mathrm{E}-09$ & $2.29 \mathrm{E}-09$ & $4.33 \mathrm{E}-09$ \\
200 & $2.79 \mathrm{E}-05$ & $3.60 \mathrm{E}-05$ & $6.55 \mathrm{E}-05$ \\
300 & $6.88 \mathrm{E}-04$ & $9.01 \mathrm{E}-04$ & $1.62 \mathrm{E}-03$ \\
400 & $3.45 \mathrm{E}-03$ & $4.47 \mathrm{E}-03$ & $8.15 \mathrm{E}-03$ \\
500 & $9.18 \mathrm{E}-03$ & $1.15 \mathrm{E}-02$ & $2.17 \mathrm{E}-02$ \\
600 & $1.79 \mathrm{E}-02$ & $2.14 \mathrm{E}-02$ & $4.24 \mathrm{E}-02$ \\
700 & $2.92 \mathrm{E}-02$ & $3.28 \mathrm{E}-02$ & $6.91 \mathrm{E}-02$ \\
800 & $4.27 \mathrm{E}-02$ & $4.47 \mathrm{E}-02$ & $1.01 \mathrm{E}-01$ \\
900 & $5.79 \mathrm{E}-02$ & $5.64 \mathrm{E}-02$ & $1.36 \mathrm{E}-01$ \\
1000 & $7.43 \mathrm{E}-02$ & $6.77 \mathrm{E}-02$ & $1.73 \mathrm{E}-01$ \\
1100 & $9.16 \mathrm{E}-02$ & $7.81 \mathrm{E}-02$ & $2.11 \mathrm{E}-01$ \\
1200 & $1.09 \mathrm{E}-01$ & $8.79 \mathrm{E}-02$ & $2.49 \mathrm{E}-01$ \\
1300 & $1.27 \mathrm{E}-01$ & $9.70 \mathrm{E}-02$ & $2.86 \mathrm{E}-01$ \\
1400 & $1.45 \mathrm{E}-01$ & $1.05 \mathrm{E}-01$ & $3.20 \mathrm{E}-01$ \\
1500 & $1.63 \mathrm{E}-01$ & $1.14 \mathrm{E}-01$ & $3.53 \mathrm{E}-01$ \\
1600 & $1.80 \mathrm{E}-01$ & $1.21 \mathrm{E}-01$ & $3.83 \mathrm{E}-01$ \\
1700 & $1.96 \mathrm{E}-01$ & $1.28 \mathrm{E}-01$ & $4.10 \mathrm{E}-01$ \\
1800 & $2.12 \mathrm{E}-01$ & $1.36 \mathrm{E}-01$ & $4.35 \mathrm{E}-01$ \\
1900 & $2.27 \mathrm{E}-01$ & $1.42 \mathrm{E}-01$ & $4.57 \mathrm{E}-01$ \\
2000 & $2.41 \mathrm{E}-01$ & $1.49 \mathrm{E}-01$ & $4.76 \mathrm{E}-01$ \\
2100 & $2.55 \mathrm{E}-01$ & $1.55 \mathrm{E}-01$ & $4.93 \mathrm{E}-01$ \\
2200 & $2.67 \mathrm{E}-01$ & $1.62 \mathrm{E}-01$ & $5.09 \mathrm{E}-01$ \\
2300 & $2.79 \mathrm{E}-01$ & $1.68 \mathrm{E}-01$ & $5.23 \mathrm{E}-01$ \\
2400 & $2.90 \mathrm{E}-01$ & $1.74 \mathrm{E}-01$ & $5.35 \mathrm{E}-01$ \\
2500 & $3.00 \mathrm{E}-01$ & $1.79 \mathrm{E}-01$ & $5.46 \mathrm{E}-01$ \\
2600 & $3.10 \mathrm{E}-01$ & $1.85 \mathrm{E}-01$ & $5.57 \mathrm{E}-01$ \\
2700 & $3.19 \mathrm{E}-01$ & $1.90 \mathrm{E}-01$ & $5.66 \mathrm{E}-01$ \\
2800 & $3.28 \mathrm{E}-01$ & $1.96 \mathrm{E}-01$ & $5.75 \mathrm{E}-01$ \\
2900 & $3.36 \mathrm{E}-01$ & $2.01 \mathrm{E}-01$ & $5.83 \mathrm{E}-01$ \\
3000 & $3.43 \mathrm{E}-01$ & $2.06 \mathrm{E}-01$ & $5.90 \mathrm{E}-01$ \\
\hline & & &
\end{tabular}

Note: In Table 7 ,

T-Temperature $\left({ }^{0} \mathrm{~K}\right)$

$[a]-A l H+H D \Leftrightarrow A l D+H_{2}$

[b] $-A l D+H D \Leftrightarrow A l H+D_{2}$

$[c]-A l H+O D \Leftrightarrow A l D+O H$ 